\title{
PLUGGY: A Pluggable Social Platform for Cultural Heritage Awareness and Participation
}

\author{
Veranika Lim ${ }^{1}$, Nikos Frangakis ${ }^{2}$, \\ Luis Molina Tanco ${ }^{3}$, and Lorenzo Picinali ${ }^{1}$ \\ ${ }^{1}$ Imperial College London, London, United Kingdom \\ v.lim@imperial.ac.uk, l.picinali@imperial.ac.uk \\ ${ }^{2}$ Institute of Computer and Communication Systems, Zografou, Greece \\ nikos.frangakiseiccs.gr \\ ${ }^{3}$ University of Malaga, Malaga, Spain \\ imtancoluma.es
}

\begin{abstract}
One of the preconditions for genuine sustainability is a heritage that is present anywhere and anytime in everyday life. We present PLUGGY, a Pluggable Social Platform for Heritage Awareness and Participation. PLUGGY will address the need of society to be actively involved in cultural heritage activities, not only as an observer but also as a creator and a major influencing factor. With PLUGGY, we aim to bridge this gap by providing the tools needed to allow users to share their local knowledge and everyday experience with others, together with the contribution of cultural institutions. Users will be able to build extensive networks around a common area of interest, connecting the past, the present and the future. It will be powered by its users and puts people's values, aspirations and needs first. Users of PLUGGY will be the providers of information about cultural heritage in the everyday and ordinary, real life. Through its social platform and by using its innovative curation tools, designed to solely focus on a niche area in social media, citizens will be able to act as skilled storytellers by creating fascinating personalised stories and share them through social networking with friends, associates and professionals. In this paper, we describe a structured formative and summative evaluative approach of PLUGGY's core concepts, which results will be used to inform and improve its design.
\end{abstract}

Keywords: Distributed Curation, Social Media, Cultural Heritage, Mobile Applications, 3D Audio, Virtual and Augmented Reality, Collaborative Games, Geolocation. 


\section{Introduction}

\subsection{A new Paradigm in Cultural Heritage}

The Faro Convention argues that a heritage that is everywhere, and relevant to everyday life, is likely to be one of the preconditions for genuine sustainability. This is certainly the case at the social and cultural levels, but also at the economic and environmental ones [9]. The convention itself stresses the importance of heritage communities, deemed as social groups who value specific aspects of cultural heritage which they wish to sustain and transmit to future generations within the framework of public action [7]. The Faro Convention outlines a framework for considering the role of citizens in the definition, decisionmaking and management processes related to the cultural environment in which communities operate and evolve. Citizen participation has become an ethical obligation and a political necessity. It revitalises society, strengthens democracy and creates governance that can renew the conditions for living together, encouraging wellbeing and a better quality of life. Thus, a new heritage paradigm is becoming visible. In the traditional view, material things were privileged, and values were based on supposedly intrinsic properties or represented a national history. This was a paradigm that encouraged the reduction of heritage to tourism and consumption. In contrast, the emerging new paradigm puts the production of heritage in the foreground, and aims to encompass greater democratic participative action, with greater concern for the local and the everyday. It uses the concept of landscape that is promoted by the European Landscape Convention (which is increasingly popular in academia and policy) as a global frame for heritage, recognising that heritage assets and objects offer fundamental social and economic values and benefits far beyond those traditionally recognised [9].

\subsection{Enablers of the Paradigm Shift}

Policy. The role of culture as a component of sustainable development is being increasingly discussed in policy debates. UNESCO emphasised the importance of culture during the Decade of Culture and Development (1988 - 1998) and through its Conventions (e.g., on the Protection and Promotion of the Diversity of Cultural Expressions in 2005; for the Safeguarding of the Intangible Cultural Heritage in 2003; and concerning 
the Protection of the World Cultural and Natural Heritage in 1972). It is currently working towards the objective of including culture in the UN Post 2015 Millennium Development Goals (UNESCO Hangzhou Declaration in May 2013). In Europe, both the Council of Europe's Landscape Convention and its Faro Convention imply more culturallysensitive approaches [9].

Inclusivity. One key approach to cultural heritage is inclusivity. Heritage is most often represented by the best buildings and monuments. Rarely are these located where most people live, 'here'. Too often they are - it seems almost by definition — somewhere else, 'there'. People might visit them on holiday, but this type of heritage is not part of the everyday landscape of their normal lives. If heritage is thus defined as elsewhere, there is a risk that it will unintentionally become an instrument of exclusion. It can, however, become an instrument of inclusion and commonality if, following Faro, it is defined contextually as local, lived-in, ordinary, if it is seen as a legacy from our predecessors rather than more narrowly from ancestors, and if it is recognised as an element of both shared identity and differentiation [15]. Furthermore, the modern accepted concept of heritage is much more extensive, including tangible, natural and intangible heritage. In 2003, following a strong debate [2], the General Conference of the Unesco established the foundations for the safeguarding of the intangible cultural heritage. As a result, Unesco extended the previously accepted definition of cultural heritage, restricted to monuments, buildings and sites and included oral traditions and expressions including language, performing arts, social practices, rituals and festive events, knowledge and practices and traditional craftsmanship. Thence, heritage is not just anywhere since it involves communities playing a central role in safeguarding activities of intangible cultural heritage. States were asked to ensure the widest possible involvement of communities and individuals in the creation, transmission and even management of such heritage. After the Faro Convention, the paradigm shift moved beyond considering inclusivity at the heart of current heritage policies. Inclusivity is now the goal, and participatory creation, transmission and management of cultural heritage are the means to that goal.

Technology. Because of careful planning of mobile technology manufacturers and operators [8], we have reached an always-connected 
society. The combination of almost ubiquitous access to multimedia content and information with the consolidation of crowdsourcing, creates great opportunities to develop technological tools to further enable this paradigm shift in cultural heritage, such as PLUGGY. Crowdsourcing is a phenomenon by which citizen communities have accepted to freely provide this content and information [13].

\section{PLUGGY}

Currently, limited ICT tools exist to provide better support to citizens in their everyday activities in shaping cultural heritage and being shaped by it. There are important initiatives to build applications and repositories for heritage dissemination which compile collections from museums, libraries and other institutions through virtualization (e.g., Europeana, Google Cultural Institute). However, these have been top-down driven by institutions and have so far not succeeded at involving citizens in the creation of heritage communities around them. In contrast, current social platforms have demonstrated their potential to build networks through the individual and distributed contributions of users. However, their possibilities have not been fully exploited with regards to cultural heritage promotion and integration in people's everyday life. PLUGGY aims to bridge this gap by providing the necessary tools to allow users to share their local knowledge and everyday experience with others together with the contribution of cultural institutions such as museums. This joint effort builds extensive networks around a common interest in connecting the past, the present and the future. This is in accordance to Flinn [10], who emphasized the importance of diversity within our national histories and archives, and that we all, professional and nonprofessionals, need to find a way of ensuring that community histories and archives are preserved.

The objectives in this project are to: (1) design, develop and implement a heritage centric social platform; (2) design an architecture of the social platform to allow the easy integration of applications; the scalability of the platform and the support of specialized devices (e.g., AR/VR/trackers etc.); (3) design, develop and implement the integration of the PLUGGY Social Platform with online digital collections and other social media; (4) design, develop and implement the curatorial tool for creating stories with meaningful narratives resulting to Virtual Exhibitions around 
specific topics; (5) design, develop and implement 4 different applications, utilizing the social platform and the curatorial tool, in order to showcase the potential of the platform and to be used to kick start applications for the after project life of the platform; and finally (6) evaluate the impact of PLUGGY and the pluggable applications in a variety of case studies.

\section{Background}

\subsection{The Role of Communities}

According to Giaccardi et al. [11], contemporary heritage studies teach us that values are not attached to just artefacts, buildings or sites. Nor are they frozen in time. Instead, they are the results of ongoing interactions in the lived world of ordinary people. Giaccardi et al. emphasizes that heritage is something we socially construct in the context of our own lives as a way of meaningfully interacting with our past and shaping our vision of the future. Fortunately, digital and social technologies are facilitating distributed forms of curatorial practice, which can be harnessed to democratize history [16]. Although, we still need to understand better whether and how ubiquitous and communication technologies like social media shape and sustain a shared sense of identity and belonging for current and future generations [11], there are examples of previous work in cultural heritage where social media plays a central role, e.g. in distributed curation and personalization (further discussed below).

\subsection{Distributed Curation}

Liu [16] describes distributed curation as a socio-technical practice involving people, cultural artefacts, and information and communication technology. It's a collaborative and distributed practice, creating shared ownership over the stewardship of the living heritage through transparency, which further allows other parties to partake in the curatorial process. One well-known social media channel, generally accepted and used by communities, is Instagram. Instagram allows creative practices from non-elite social contexts and communication that relies on everyday competencies rather than a formal artistic education (Burgess, 2006 as described in [19]). In making selections as to what to 
display and which narrative to tell, Instagrammers somehow act as virtual curators (Hogan, 2010 as described in [19]), extending the reach of the museum beyond its walls [19]. In supporting rural community heritage, the CURIOS project [18] explored how digital archives for rural community heritage groups can be made more sustainable so that volunteer members can maintain a lasting digital presence. It was developing software tools to help remote rural communities to collaboratively maintain and present information about their cultural heritage using open linked data. This approach is attuned to the specificity of a local heritage but can also take advantage of already collected materials from elsewhere. Yelmi et al. [20] evaluated soundscapes as an intangible cultural heritage element and introduced the Soundsslike Project; a crowdsourced online sound archive which invites people to record symbolic urban sounds and upload them to the online sound archive. This online platform was built and displayed in an exhibition by means of an interactive table top interface to learn more from users and contributors, and to enrich the archive content by raising public awareness of urban sounds.

\subsection{Personalization in Cultural Encounters}

Social media can also play a role in the personalization of information technologies. People are often overloaded with an increasing amount and variety of cultural items making it difficult to identify what is interesting. Therefore, there is a need to personalize visits to cultural objects, to visitors' knowledge and connections, to ensure interactions are effective. For example, ArtLinks was developed to provide a guidance system based on a public display in museum exhibits that allowed visitors to create and use tags to help guide other visitors [5]. ArtLinks aimed at encouraging social interactions and enhancing experiences by supporting visualization of people, words and their connections related to an exhibition. Similarly, MobiTag [6] is an electronic guide that supports semantic, social, and spatial navigation in museums by allowing visitors to create and vote for tags. Furthermore, Han et al. [12] developed a mobile application called Lost State College (LSC) and showed that users utilized social features as a way of learning local history and interacting with others, co-creating digital traces and rich layers of local history information. Users shared information using social features, which allowed different types of connection to the local history. 
Personalization, derived through interactions between visitors of cultural environments, has also been supported using data from popular available social media sources such as Twitter, Instagram, Wikipedia and Flickr. For example, McGookin and Brewster designed PULSE [14] to allow users to gain a vibe (i.e., an intrinsic understanding of the people, places and activities around their current location) using Twitter data. As users moved, PULSE downloaded public messages (tweets) generated by any user in the current location. Then, PULSE would select the closest tweet and insert it in a virtual 3D auditory environment: users heard tweets as whispered conversations. Bellens et al. [1] explored how social media data can be employed to study tourism on European Cultural Routes and showed its potential for investigating a complex touristic object such as a cultural route. They combined text related to photos on Instagram with Wikipedia for geographical places. This allowed them to identify the most popular stops and localities related to the cultural route. Bujari et al. [4] proposed PhotoTrip, an interactive tool able to autonomously recommend cultural heritage locations along travel itineraries even if those locations were not mainstream. PhotoTrip identified points of interest by gathering pictures and related information from Flickr and Wikipedia and then providing the user with suggestions and recommendations.

Related work has demonstrated how digital cultural heritage transforms the way of experiencing or learning community heritage through social media. The community are being more and more involved through distributed curation, i.e., where technologies mediates and allows them to contribute to our histories, and personalization, i.e., where communities' online activities are used to aid in decision-making. Current social platforms have demonstrated their potential to build networks through the individual and distributed contributions of users. To our knowledge, however, their possibilities have not been fully exploited with regards to cultural heritage promotion and integration in people's everyday life.

\section{Methodology}

\subsection{PLUGGY Technologies}


The Social Platform. PLUGGY will be built around a heritage-centric social platform ensuring that citizens are at the heart of PLUGGY. The social platform will be the place where citizens, heritage communities and professionals are able to share their curated Virtual Exhibitions, visit Virtual Museums and browse the Digital Collections created by others. Users of the social platform will upload, tag, categorize and describe assets in the form of high quality images and videos, text, 3D models and $3 \mathrm{D}$ audio that will be experienced through the pluggable applications. The platform will interface with Europeana, the British Museum's Collection OnLine and other existing online Digital Collections to allow citizens to experience and relate tangible, intangible and natural heritage collections through PLUGGYs browsing functionalities. The social platform will deploy artificial intelligence methods for the semantic tagging of its content and the Virtual Exhibitions, facilitating also an automated way of creating Virtual Museums by grouping Virtual Exhibitions. See figure 1.

The Curatorial Tool. The Curatorial Tool will allow users to create Virtual Exhibitions hosted on the social platform. The Curatorial Tool allows the curation of stories, which link the digital collections uploaded within the social platform and those available through the interfaces to the Digital Collections. Users of PLUGGY curate content that can be accessed using novel interfaces (Augmented Reality, Geolocation), and thus experience stories in new engaging ways. The Curatorial Tool has an open architecture that allows for development of future applications with interfaces which have not yet been developed.

Pluggable Applications. Through the Pluggable Applications, the users experience the stories created and hosted on PLUGGY's social platform. Applications have two sides to them: on the one hand, they allow users to experience the content of the Virtual Museums in novel and engaging ways. On the other, they extend the Curation Tool through their curation application interfaces. The Pluggable Applications can be combined for a rich multi-sensory experience. A total of 4 applications showcase the potential of PLUGGY's content.

Augmented Reality Application. This application allows stories to be experienced through virtual models and overlaid information, on live video streams. The application authoring interface extends the Curation 
Tool to allow association of assets and behaviours to markers for augmenting real exhibitions and other experiences with virtual content.

3D Audio Application. The 3D Audio application allows for the creation and interactive playback of 3D sonic narratives and, more generally, the creation of 3D audio content within PLUGGY. In addition to a 3D sound spatialization tool (both for loudspeakers and headphone

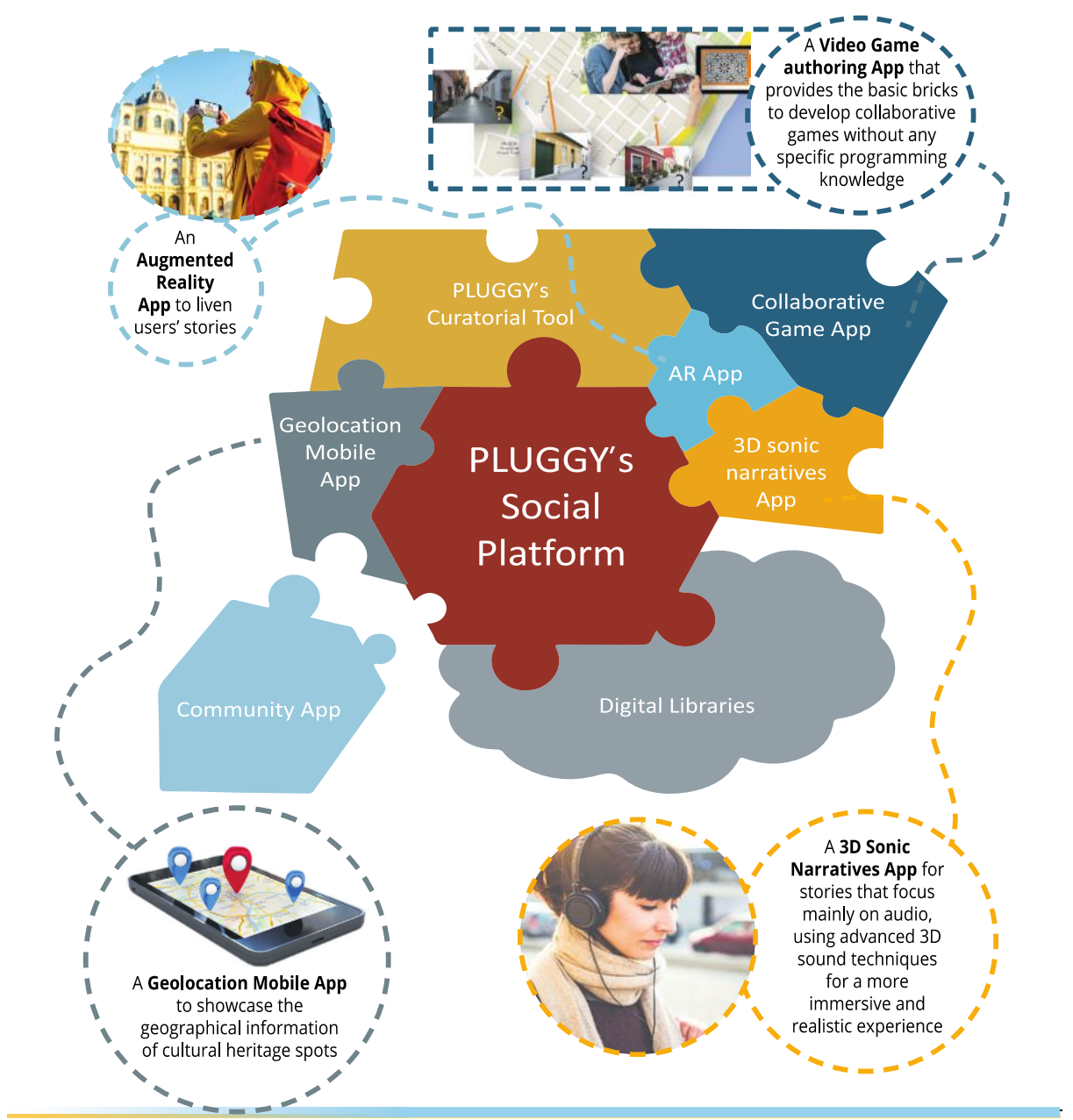

Fig. 1. PLUGGY's concept structure of the social platform, curatorial tool and pluggable applications.

playback), the application will integrate functions/modules for selecting, 
editing and mixing audio from existing sources (e.g., import audio-les), and for applying sound effects (e.g., reverberators, spatial spread algorithms, etc.).

Collaborative Game Application. The Collaborative Game application allows for video game authoring by providing the basic bricks (i.e., rules, routes) to develop collaborative games. This application does not require programming knowledge and enables participants to engage in asset and story discovering through challenges and other gamification-based engagement techniques.

Geolocation Application. The Mobile Geolocation application targets outdoor activities. With this application, geo-located stories are made available to users when they are physically near the coordinates of assets where they are virtually situated. The application extends the Curation Tool by allowing curators to situate virtual content geographically on a map at specific coordinates.

\subsection{User-Centred Design Approach}

The development of PLUGGY takes a user-centred design approach around several case studies and user evaluations in the field. It is of high importance for the concept to be adapted to and evaluated through different case studies to account for a variety of contexts of use to inform its final design. Although, prototypes of PLUGGY will also be evaluated through custom usability tests and expert reviews to evaluate its main functionalities, this concept paper mainly discusses the planned field studies in the formative and summative evaluation stages.

Formative evaluation. The first stage contains an exploration on PLUGGY's Core Concepts.

Research Objectives. In this first stage, we aim at (1) broadening the narrative scope of artefacts, i.e., by connecting physical artefacts with digital artefacts in a mixed media environment, and connecting people and stories through distributed curation. We further aim at (2) exploring the impact of distributed content curation on people's engagement and participation in cultural heritage. We also aim at (3) creating and promoting a more immersive approach to distributed curated artefacts by 
adding features e.g., 3D audio and Virtual Reality, and (4) exploring its impacts on visitor's engagement and participation in cultural heritage. Finally, we aim at (5) understanding commonalities and/or differences between socio-demographic groups.

Case Studies. We will select 2 case studies: a typical museum site and an outdoor historical site. For the museum site, we will select a gallery that displays a variety of everyday artefacts. Daily artefacts are expected to invite narratives contributable by the public. For the second site, we will select an architectural collection, which are usually very popular tourist attractions. They can be used as an artefact to promote ethics, history, or industry, but can be experienced by visitors through a narrow understanding of time and place. Architecture can be an immersive space into the city's history, but people often lack knowledge about it. An interesting aspect is that it can be an environment shared by a mixed public including tourists, employees, and locals, while limited information is available to contextualize the artefacts.

Study Design. For each site, 3 to 4 artefacts will be selected for the study. The artefacts will be exhibited in different display design conditions, e.g., each display design is presented for a week:

Condition 1: the artefacts are presented with the current curator's information only. This forms the baseline from which evaluations will be made.

Condition 2: the artefacts are presented with the current curator's information as well as visitor's curated content using Instagram or Medium centralized into a website. Visitors will be triggered with open questions such as: "What does this artefact/building remind you of?", "What influences do you see?", and "Can you identify any specific artistic/architectural features?".

- Curating stories: Visitors will be asked to use Instagram or Medium for sharing their answers or stories. They will be asked to use one specific hashtags (for Instagram) and tags (for Medium), for the study project and artefact. Stories shared on Medium will be accessible through a custom WordPress website (one per case study). Each website will contain a link to each 
artefact and each artefact page will have a list of links to shared stories in Medium on the specific artefact). Participants' own devices can be used to curate information.

- Accessing stories: Answers and stories shared using Instagram, will be accessible by using the specific hashtag with the Instagram application. Answers and stories shared in Medium will be accessible through the custom WordPress website using a QR code or a link. Participants' own devices can be used to access information.

Condition $3 \mathrm{a}$ and $\mathrm{b}$ : each artefact page will contain 3D audio narratives of selected 'quotes' from the different stories and/or 3D soundscapes.

Audio narratives and soundscapes are accessible on visitors own devices as well as on a tablet publicly available.

Condition 4: each artefact contains a 3D virtual image accessible on visitors own devices as well as on the tablet publicly available.

Technological equipment. To conduct the study, the following equipment will be used: (1) laminated information labels with the questions, hashtags and tags, a QR code and a link to the project website. A video camera will be used to record interactions. 3 tablets will be made available for those who do not wish to use their own devices. These tablets will have Wi-Fi access and will be secured on a standalone tablet stand near each artefact. Each tablet will include acoustic speakers and custom earplugs for 3D augmented audio. One tablet will be used for post questionnaires.

Procedures. For each condition described above, visitors will be shadowed and observed during their interactions with the artefacts and available digital information. A map of the test site will be used to annotate navigation, touch points, and note behavioural insights. In a post-test measurement, upon exiting the site, visitors will be invited to participate in an online questionnaire and sign informed consents. Visitors will also be asked general information such as their demographics, where they came from, what brought them to the site, where they are going, what they know about the site, what they would like to know about the site, about their everyday technology use, and 
their social media use. They will be given an information sheet for a debriefing on the objectives of the study.

Study outcome measures. The study will provide insights on the narrative scope of artefacts and will underline to what extent distributed content curation have an impact on visitor engagement. Engagement is measured using the standardized MEC spatial presence questionnaire [17], through structured observations, and online activities. The study will also provide insights into the additional impact of 3D audio and virtual reality on visitor engagement and participation in cultural heritage. Findings from this stage will be used to inform the initial design of PLUGGY which will undergo a summative evaluation.

Participants entry. Visitors will be triggered with a link to the displays, which they can voluntarily use. Upon exiting the site, visitors will be approached to fill out the questionnaires and informed consent form. A random and opportunity sampling technique will be applied. Visitors will not be obliged to participate and can opt out of participating in the study at any time. For the video-recorded observations, all individuals will be anonymized by blurring facial images.

Data analysis. Collected data will be compared between the display conditions. A thematic analysis will be performed on the stories and pictures posted in Instagram and Medium. A quantitative analysis will be performed on the observed behaviours around the artefacts. Data from questionnaires will be analysed quantitatively and thematically.

Summative Evaluation. The second stage contains an exploration on the user experience around PLUGGY's final technologies.

Research Objectives. In this stage, we aim at (1) exploring the use, usability, and impact of PLUGGY's social platform, curatorial tool and mobile applications on people's engagement and participation in cultural heritage. We also aim at (2) understanding commonalities and/or differences between socio-demographic groups.

Case Studies. We will select a number of case studies, including typical museum sites, outdoor historical sites, and individuals with tangible and intangible collections not necessarily related to specific sites. In addition 
to the selected case studies in the formative evaluation, we will involve the museums as part of the project, which are the Open-Air Water Power Museum in Greece, the Environment Museum in Greece, the Museum of Silversmithing in Greece, and the East Slovakian Museum in Slovakia. These case studies will be run for a period of at least 6 months.

Study Design. For each site, 3 to 4 artefacts will be selected for the study. The artefacts will be displayed in different display design conditions:

Condition 1: the artefacts are presented as is with the curator's information only. This will form the baseline against which comparisons can be made.

Condition 2: the artefacts are presented as is with the curator's information, as well as visitor's curated content when using PLUGGY's Social Platform. The public will be able to curate stories using the Curatorial Tool. For each artefact, the suite of mobile applications can be used for 3D audio narratives and soundscapes, 3D augmented reality, geolocation (only for outdoor test sites), and collaborative games. Visitors in both museum and historical sites and individuals with tangible and intangible collections not necessarily related to specific sites will be asked to use PLUGGY's technology in any way they wish.

Technological equipment. To conduct the study in the selected museums and historical sites, the following equipment will be used: (1) laminated information labels with hashtags and tags, and a QR code linking to the study project's website. A video camera will be used to record interactions. Optionally, 3 tablets will be made available for those who do not wish to use their own devices. These tablets will have Wi-Fi access and will be secured on a standalone tablet stand near each artefact. Each tablet will include acoustic speakers and custom earplugs for 3D audio. One tablet will be used for post questionnaires. Individuals with tangible and intangible collections not necessarily related to specific sites will be using PLUGGY's technologies on their own devices.

Procedures. The same procedures as in the formative evaluation will be applied for the selected test sites. The following procedures apply to individuals with tangible and intangible collections not necessarily related to specific sites. First, the participant will be given an information 
sheet on the objectives and procedures of the study and an informed consent form. They will be asked to use the PLUGGY in any way they wish. Intermittently and in a post-test measurement, individuals will be invited to participate in online questionnaires.

Study outcome measures. The study will provide insights in the narrative scope of artefacts and to what extend PLUGGY's technologies have impact on visitor's engagement and participation in cultural heritage. Engagement and participation is measured using the standardized MEC spatial presence questionnaire (relevant only for the test sites) [17], experience narratives, through structured observations (relevant only for the test sites), and online activities. Other questionnaires aim at collecting users' positive and negative experiences of specific functionalities and at exploring perceived usability of the technologies using the System Usability Scale (SUS) [3]).

Participants entry. Museum and historical site visitors will be asked to voluntarily participate upon entering and exiting the site. A random and opportunity sampling technique will be applied. Visitors will not be obliged to participate. They can refuse participation or withdraw anytime without repercussions. Individuals will be recruited on site, and through professional and social networks.

Data analysis. PLUGGY's usage will be analysed through descriptives. A quantitative and qualitative analysis will be performed on the online and offline (i.e., observations relevant only for the test sites) interactions between users of PLUGGY. A quantitative analysis will be performed on the data from the questionnaires. Thematic analysis will be performed on the experience narratives.

\section{Conclusion, Challenges and Impacts}

With PLUGGY, we aim to transfer the responsibility towards cultural heritage from the exclusive domain of experts to individuals and heritage communities, giving them the opportunity to expose and transmit their values to anyone sharing their interests, visions and concerns. With PLUGGY's social platform, Curatorial Tool and pluggable applications, any sensitized individual will be able to enrich the cultural heritage of their focal point by uploading materials (i.e. audio, video, images, text, 
3D models) and use these in combination with what is already available in the platform to create a more personalized, interactive, and to-thepoint story which can then be shared online. PLUGGY, however, will face several challenges. For example, is there room for yet another social platform? And what is the value of having a centralized social platform specifically for cultural heritage over the potential of the various social media services already in use? Moreover, it might be argued that PLUGGY also faces the challenges inherent to the paradigm shift in cultural heritage [2]; institutions and professionals trained to safeguard traditional cultural heritage may have difficulties in applying their skills to safeguard intangible heritage (or any other type of heritage) due to differences in perceptions or truths held in society. On the other hand, it will allow for a diversity of ways to look at history. This will further extend to the issue of trust. What mechanisms should be implemented to gain trust in content and content creators for an effective use of a platform like PLUGGY? Nevertheless, we expect PLUGGY to have significant impacts socially, economically and ecologically. First, it can promote wider understanding of heritage. Second, it can improve innovation capacity and integration of new knowledge as it will mobilize the economic sectors, i.e., tourism and the creative industries, indirectly promoting local development and entrepreneurship. Finally, it is expected to promote cultural diversity.

\section{Acknowledgement}

This work is a part of the PLUGGY project. PLUGGY has received funding from the European Union's Horizon 2020 Research \& Innovation Programme under grant agreement no 726765. Content reflects only the author's view and European Commission is not responsible for any use that may be made of the information it contains.

\section{References}

1. Bellens A., Grabar, N., Valmond Le Banc, N., Kergosien, E., Eloire, F and Severo. M.: Social Media and European Cultural Routes: Instagram Networks on the Via Francigena. In: The International Conference on Management of Computational and Collective Intelligence in Digital EcoSystems, pp. 122 - 128. ACM, New York, USA (2016).

2. Bortolotto, C: Le Trouble Du Patrimoine Culturel Immatériel, Terrain (2011).

3. Brooke, J.: SUS: A "quick and dirty" usability scale". In: Jordan, P. W., Thomas, B. A. Weerdmeester and McClelland, I. L. (eds) Usability evaluation in industry, pp. 189194. Taylor \& Francis, London (1996). 
4. Bujari, A., Ciman, M., Gaggi, O., and E. Palazzi, C.: Using gamification to discover cultural heritage locations from geo-tagged photos. In: Personal and Ubiquitous Computing, pp. 235 - 252. Springer-Verlag, London, UK (2017).

5. Cosley, D., Lewenstein, J., Herman, A., Holloway, J., Baxter, J., Nomua, S., Boehner, K., and Gay, G.: ArtLinks: Fostering Social Awareness and Reflection in Museums. In: Proceedings of the SIGCHI Conference on Human Factors in Computing Systems, pp. 403 - 412. ACM, New York, USA (2008).

6. Cosley, D., Baxter, J., Lee, S., Alson, B., Nomura, S., Adams, P., Sarabu, C., and Gay, G.: A Tag in the Hand: Supporting Semantic, Social, and Spatial Navigation in Museums. In: Proceedings of the SIGCHI Conference on Human Factors in Computing Systems, pp. 1953 - 1962. ACM, New York, USA (2009).

7. Council of Europe Treaty Series - No. 199, https://rm.coe.int/1680083746, 2005, last accessed 2017/06/01.

8. Cushing, B.: Always Connected. Beyond Broadband - What Comes Next? In: Broadband Services, Applications, and Networks: Enabling Technologies and Business Models, pp. 15 - 23. Professional Education International Inc., Chicago, USA (2011).

9. Fairclough, G., et al.: The Faro Convention, a New Paradigm for Socially - and Culturally - Sustainable Heritage Action? In: Култура/Culture, pp. 9 - 19. (2014).

10. Flinn, A..: Community Histories, Community Archives: Some Opportunities and Challenges, 20(2), 151 - 176 (2007).

11. Giaccardi, E., F. Churchill, E., and B. Liu, S.: Heritage Matters: Designing for Current and Future Values Through Digital and Social Technologies. In: Extended Abstracts on Human Factors in Computing Systems. pp. 2783 - 2786. ACM, New York, USA (2012).

12. Han, H., Shih, P. C., Rosson, M. B., and Carroll, J. M.: Enhancing Community Awareness of and Participation in Local Heritage with a Mobile Application. In: Proceedings of the 17th ACM conference on Computer Supported Cooperative Work \& Social Computing, pp. 1144 -1155. ACM, New York, USA (2014).

13. Howe, J.: The Rise of Crowdsourcing. In: Wired Magazine (2006).

14. Mcgookin, D., and Brewster, S.: PULSE: An Auditory Display Provide a Social Vibe. In: Proceedings of Interacting with Sound Workshop: Exploring Context-Aware, Local and Social Audio Applications, pp. 12 - 15. ACM, New York, USA (2012).

15. Wolferstan, S., and Fairclough, G.: Common European heritage: reinventing identity through landscape and heritage? In: Callebaut, D., Mařík, J; Maříková-Kubková, J. (eds) Heritage Reinvents Europe, EAC Occasional Papers, pp. 43 - 54. European Archaeological Council / Archaeolingua, Budapest, Hungary (2013).

16. Liu. S. L.: Grassroot Heritage: A multi-method Investigation of how Social Media Sustain the Living Heritage of Historic Crises. PhD thesis, (2011).

17. Vorderer, P., Wirth, W., Gouveia, F. R., Biocca, F., Saari, T., Jäncke, F., Böcking, S., Schramm, H., Gysbers, A., Hartmann, T., Klimmt, C., Laarni, J., Ravaja, N., Sacau, A., Baumgartner, T. \& Jäncke, P.: MEC Spatial Presence Questionnaire (MECSPQ): Short Documentation and Instructions for Application. Report to the European Community, Project Presence: MEC (IST-2001-37661). Online. Available from http://www.ijk.hmthannover.de/presence, (2004).

18. Webster, G., Nguyen, H. H., Beel, D. E., Mellish, C., Wallace, C. D., and Pan, P.: CURIOS: Connecting Community Heritage through Linked Data. In: Proceedings of the 17th ACM conference on Computer Supported Cooperative Work \& Social Computing, pp. 639 - 648. ACM, New York, USA (2015).

19. Weilenmann, A., Hillman, T., and Jungselius, B.: Instagram at the Museum: Communicating the Museum Experience through Social Photo Sharing. In: Proceedings of 
the SIGCHI Conference on Human Factors in Computing Systems, pp. 1843 - 1852. ACM, New York, USA (2013).

20. Yelmi, P., Kuscu, H., and Yantac, A. E.: Towards a Sustainable Crowdsourced Sound Heritage Archive by Public Participation: The Soundsslike Project. In: Proceedings of the 9th Nordic Conference on Human-Computer Interaction, 71. ACM, New York, USA (2016). 